\title{
Polyelectrolyte determination in drinking water
}

\author{
S Majam* and PA Thompson \\ Umgeni Water, PO Box 9, Pietermaritzburg 3200, South Africa
}

\begin{abstract}
Chemical contaminants that occur in drinking water are not usually associated with acute health effects when compared to microbial contaminants and are usually given a lower priority. Those that are of concern have cumulative toxic properties such as metals and substances that are carcinogenic. Some of these potentially hazardous chemical contaminants are a consequence of the treatment chemicals themselves e.g. organic polyelectrolytes used as coagulant aids in water treatment. The presence of residues of the un-reacted monomer in these polyelectrolyte products is a cause for concern.

Historically, inorganic coagulants such as aluminium sulphate and ferric chloride have been used as coagulants/flocculants in the treatment of drinking water. The residual amounts of these chemicals were easy to detect and to control using readily available standard methods. The increasing use of polyelectrolytes has created a problem for the potable water industry as there are no readily available methods for the determination of residual polyelectrolyte concentration.

This study aims at extending existing analytical techniques and comparing them to determine results that are most accurate and reliable to the quantification of residual polyelectrolytes.
\end{abstract}

Keywords: polyelectrolytes, chemical contaminants, health effects, analytical techniques

\section{Introduction}

Polydiallylydimethyl ammonium chloride (polydadmac) and epichlorohydrin-dimethylamine (epi-dma) are established coagulants in the treatment of drinking water. Their efficiency can be seen in the fact that approximately $75 \%$ of waterworks in South Africa have adopted these polyelectrolytes as part of their water treatment process (Leopold, 2004).

Polyelectrolyte products used in the water supply industry may contain in addition to polyelectrolyte, measurable amounts of certain contaminants. These contaminants are essentially unreacted raw material from the polyelectrolyte manufacturing process, e.g. the monomers, un-reacted chemicals used to form the monomer units, initiators, quenchers, etc. A list of contaminants that may be found in polydadmac and epi-dma are highlighted in Table 1.

Different reactants and manufacturing processes can be used to prepare what is essentially the same polymer. Process monitoring and control is therefore an important consideration in polyelectrolyte manufacture if contaminant levels are to be managed. However, national standards and regulations governing the quality of the polyelectrolyte product is something South Africa lacks (Freese et al., 2002).

Letterman and Pero (1990) have suggested that certain of these contaminants could have an adverse effect on the health of water consumers. Polyelectrolytes and their contaminants may also react with treatment chemicals added from other water treatment processes like ozonation and chlorination to form undesirable by-products (Mallevialle et al., 1984).

This is highlighted by the fact that low concentrations of polyelectrolyte remains in the water after the filtration stage and

This paper was originally presented at the 2006 Water Institute of South Africa (WISA) Biennial Conference, Durban, South Africa, 21-25 May 2006.

* To whom all correspondence should be addressed.

皿+2731 203-3009; fax:+2731 261-7202;

e-mail: Sameera.majam@umgeni.co.za

\begin{tabular}{|l|l|}
\hline \multicolumn{2}{|c|}{$\begin{array}{c}\text { TABLE 1 } \\
\text { List of contaminants found in polyelectrolyte } \\
\text { products }\end{array}$} \\
\hline Contaminant & Polyelectrolyte \\
\hline Diallyldimetylammonium chloride & Polydadmac \\
\hline Dimethylamine & Polydadmac/epi-dma \\
\hline Allylchloride & Polydadmac \\
\hline Diallylether & Polydadmac \\
\hline 5-Hexanal & Polydadmac \\
\hline Epichlorohydrin & Epi-dma \\
\hline Glycidol & Epi-dma \\
\hline 1,3-dichloro-2-propanol & Epi-dma \\
\hline 2, 3-dichloro-1-propanol & Epi-dma \\
\hline 3 chloro-1,2-propanediol & Epi-dma \\
\hline $\begin{array}{l}\text { 2-hydroxy-3-dimethylaminopropyl } \\
\text { chloride }\end{array}$ & Epi-dma \\
\hline 1,3-Bis(dimethylamino)-2-propanol & Epi-dma \\
\hline
\end{tabular}

(Source: Letterman and Pero, 1990)

continued exposure to low concentrations of contaminants such as epichlorohydrin is an important concern as it is an animal carcinogen (WHO, 1996). The techniques available for measuring residual organic polyelectrolytes in potable water are inadequate, making the need to quantify them more critical (Fielding, 1999).

\section{Review and investigation of existing analytical methodologies}

A number of methods have been devised for the quantitative determination of polyelectrolytes in water. Some of these include: colloidal titration, extraction-spectrophotometry, chromatography, fluorometry and potentiometry.

Methods that are simple to perform and that allow waterworks operators to achieve precise results are desirable as quick 
decisions and actions are sometimes required in the environmental field.

The objectives of this study were to:

- Elucidate the advantages and disadvantages of selected nonspecific analytical techniques applied to the quantification of residual polymers and

- Extend these existing analytical techniques to obtain accurate and reliable results

The objectives were achieved by subjecting water containing the polyelectrolytes, polydadmac and epi-dma to the analysis as described in the original method and its performance gauged in relation to these polyelectrolytes. Thereafter, various experimental parameters were investigated to improve the efficiency of the method as well as to determine possible interferences. Analyses were conducted on both distilled and real water systems.

The methods investigated in this study were reported as having excellent sensitivity to measure trace amounts of polyelectrolyte and are composed of simple and easy operations. They are:

\section{Potassium polyvinyl sulphate method}

This colloidal titration has been demonstrated to be an excellent method for determining the concentration of natural and synthetic polyelectrolytes (Wang et al., 1978). The authors successfully applied a colloidal titrimetric method of analysis for the determination of polyelectrolytes in wastewater. The titration method is based on a colour change in the toluidine blue indicator. Initially, the cationic polyelectrolyte (analyte) and potassium polyvinyl sulphate (titrant) form a preferential complex or colloid. When all of the analyte has been consumed, the excess titrant reacts with the indicator resulting in a colour change from blue to blue-violet. This colour change is then measured spectrophotometrically.

\section{Ponceau S dye method}

Parazak and colleagues (1987) investigated a spectrophotometric method involving the complexation of the polyelectrolyte with an anionic dye. The cationic polyelectrolyte (analyte) forms an insoluble complex when mixed with a fixed concentration of the dye. The polycation-dye complex is insoluble in water and the organic solvent. The change in the light absorbance of the dye in the aqueous phase before and after complexation is then measured. This change in absorbance is proportional to the mass of the polyelectrolyte that has complexed with the dye.

\section{Tannic acid method}

This spectrophotometric determination of the polymer is based on the complexation of the polymer with tannic acid. Since tannic acid solution is a negatively charged colloid, it is considered to form ionic bonds with cationic flocculants and hydrogen bonds with non-ionic flocculants. The transmittance measurements were then recorded at $554 \mathrm{~nm}$ and $830 \mathrm{~nm}$ respectively. Hanasaki and co-workers (1985) have demonstrated this method to be effective in quantifying trace amounts of polymer in wastewater.

\section{High-pressure liquid chromatography (HPLC)}

\section{Normal phase HPLC}

The method evaluated here was recommended for the determination of quaternary ammonium compounds in environmental matrices (HMSO, 1996). Separation of the cationic polyelectrolyte from the matrix was achieved by normal phase HPLC using a refractive index detector and quantification of the polyelectrolyte by peak height integration.

\section{Size exclusion chromatography (SEC)}

SEC investigations of both polymers were examined by using a gel filtration column. The polyelectrolyte samples were analysed in aqueous solutions using water as the mobile phase and an evaporative light scattering (ELS) detector. Nitrogen $\left(\mathrm{N}_{2}\right)$ gas was used as the nebulising gas.

\section{Results and discussion}

From the methods investigated, the first two methods were most successful in quantifying the amount of residual polyelectrolyte. These methods showed good precision with linear calibration curves.

The colloidal titration method proved to be a simple and cost effective method with minimal interference compared to the ponceau $\mathrm{S}$ dye method. The automation of analyses was crucial as it enabled bench analyses to be carried out more efficiently and rapidly and with better precision. The adaptation of the titration to a photometric titration meant that more dilute solutions could be analysed and a degree of background absorbance or turbidity can be tolerated.

The ponceau S dye method, although successful, was subject to interferences from other constituents in the sample viz. calcium, magnesium, iron and manganese. These may be obviated by prior treatment of the sample which is time consuming and necessary and may prove to be a disadvantage since quick decisions and actions are sometimes required.

The investigations using tannic acid method showed broad transmittance readings across the spectrum with no proper peaks from which a calibration curve could be constructed. The stability of sample solutions, variations in $\mathrm{pH}$, ionic strength, tannic acid concentration as well as the polyelectrolyte concentration did not provide any tangible results.

Tannins are oligomeric compounds that are derived from plant parts i.e. bark, wood, fruit, fruit pods, leaves, roots, and plant galls. They are multiple structure units with free phenolic groups and with molecular weights ranging from 500 to $>20000$. The tannic acid product provided by the manufacturer may have been inconsistent with that of Hanasaki et al. (1985) and may have contributed to the lack of sensitivity of the method to quantify polydadmac and polyamine. In addition, the large molecular weights of both tannic acid and the polyelectrolytes may have created steric hindrance, thus preventing complexation from occurring.

Normal phase high-pressure liquid chromatography (HPLC) determinations of both polymers using a refractive index detector were unsuccessful. The lack of sensitivity prompted further investigation of numerous variables including flow rate and the polarity of the mobile phase. The variables examined did not provide any single component peaks that could be used for the determination of either polymer.

HPLC using a size exclusion column and an ELS detector was able to identify both polyelectrolytes. A variation of polymer concentration and flow rate was conducted. A slower flow rate produced a well resolved single component peak. Lower concentrations of polyelectrolyte did not produce well resolved peaks. The limits of detection were $4300 \mathrm{mg} / \ell$ and $3130 \mathrm{mg} / \ell$ for polydadmac and epi-dma respectively. 


\section{Conclusion}

The quantification and identification of polydadmac and epi-dma was accomplished by the KPVS, ponceau S and SEC studies. The tannic acid method and normal phase HPLC were unsuccessful at quantifying both polyelectrolytes. Although the former two methods were subject to interference from other components present in the raw water matrix, they may be overcome by prior treatment. These methods are also operator-friendly and can be performed at a treatment works on a daily basis.

SEC studies were excellent at identifying the polyelectrolytes. The technique is highly sophisticated and must be conducted in a laboratory with trained personnel. The detection limits are practically impossible on any given occasion for a treatment works; however, in the event of an overdose or an accidental spill, it is an excellent tool to quantify the polymers.

\section{Acknowledgements}

The following institutions are acknowledged for their assistance:

- Water Research Commission for funding this project

- Umgeni Water Laboratories for their support and co-funding of the research

- Mangosuthu Technikon for use of their HPLC machine and RI detector

- Durban University of Technology for use of their HPLC machine and ESD detector.

\section{References}

FIELDING (1999) Analytical Methods for Polymers and their Oxidative By-products. AWWARF, Denver, Colorado.

FREESE S D, NOZAIC DJ and TROLLIP D L (2002) Evaluation of process chemicals. WISA Biennial Conference. Durban, South Africa. 19-23 May 2002, Durban.

HANASAKI T, OHNISHI, NIKAIDOH A, TANADA S and KAWASAKI K (1985) Determination of trace polymer in waste water. Bull. Environ. Contam. Toxicol. 35 476-481.

HIS MAJESTY'S STATIONARY OFFICE (HMSO) BOOKS (1996) Determination of Long Alkyl Chain Quaternary Ammonium Compounds in Environmental Matrices by High Performance Liquid Chromatography. London. ISBN 0117532614.

LEOPOLD P (2004) Personal communication. National Sales Manager, Sud-Chemie.

LETTERMAN RD and PERO RW (1990) Contaminants in Polyelectrolytes Used in Water Treatment. AWWARF, Denver, Colorado.

MALLEVIALLE J, BRUCHET A and FIESSINGER F (1984) How safe are organic polymers in water treatment? J. AWWA Res. Technol. $\mathbf{7 6}$ (6) 431-436.

PARAZAK DP, BURKHARDT CW and McCARTHY KJ (1987) Determination of low levels of cationic polyelectrolytes in water. Anal. Chem. 59 1444-1445.

WANG LAWRENCE K, WANG MU HAO AND KAO JAO-FUAN (1978), Application and determination of organic polymers. Water Air Soil Pollut. 9 337-348.

WHO (1996) Guidelines for Drinking Water Quality Vol. 2 (2 ${ }^{\text {nd }}$ edn.). Geneva. 